\title{
Effects of convection instability due to incompatibility between ocean dynamics and surface forcings
}

\author{
W. Cai ${ }^{1}$, P. C. $\mathrm{Chu}^{2}$ \\ ${ }^{1}$ CSIRO Division of Atmospheric Research, Aspendale, Victoria, Australia \\ 2 Department of Oceanography, Naval Postgraduate School, Monterey, CA 93943, USA
}

Received: 23 July 1996 / Revised: 20 February 1997 / Accepted: 27 February 1997

\begin{abstract}
The study demonstrates that an incompatibility between a surface temperature climatology and a given ocean model, into which the climatology is assimilated via Haney restoration, can cause model ocean climate drift and interdecadal oscillations when the ocean is switched to a weaker restoration. This is made using an idealized Atlantic Ocean model driven by thermal and wind forcing only. Initially, the temperature climatology is forcefully assimilated into the model, and an implied heat flux field is diagnosed. During this stage any incompatibility is suppressed. The restoring boundary condition is then switched to a new forcing consisting of a part of the diagnosed flux and a part of the restoring forcing in such a way that at the moment of the switching the heat flux is identical to that prior to the switching. Under this new forcing condition, the incompatibility becomes manifest, causing changes in convection patterns, and producing drift and interdecadal oscillations. The mechanisms are described.
\end{abstract}

\section{Introduction}

It is an open question as to whether or not an ocean general circulation model (OGCM) with prescribed physics is able to maintain a given set of prescribed surface climatology. In areas characterized by sharp oceanic fronts and high convective activity, the OGCM, due to the limitation of model dynamics, cannot maintain the climatology. Therefore, in these areas, the OGCM will systematically bias away from the climatology when it is allowed to do so. We refer to this situation as an incompatibility between the climatology and the OGCM dynamics. In ocean modeling, the climatology is often assimilated into an OGCM via a

Correspondence to: W. Cai
Haney (1971) restoration. This procedure effectively prescribes the surface model solution to be more or less the same as the climatology, disregarding whether or not an incompatibility exists. When such an incompatibility exists it is suppressed. Once it is allowed to manifest itself, for example, switching from a strong restoration to a weak one, or to a diagnosed flux forcing, what are the effects of the incompatibility? This is the central issue of this study. This is examined in a pure thermal circulation system. We demonstrate that it will cause a drift from the state obtained by the strong restoration and internal variabilities.

Recently, there has been a surge of research interest in the internal variability of the ocean thermohaline circulation. Kushnir (1994) presented evidence showing oceanic variabilities in the North Atlantic with a 30-40 year time scale. In a similar study, Deser and Blackmon (1993) proposed that the general warming trend in the North Atlantic sea surface temperature (SST) during the 1920s-30s and the cooling in the 1960s is associated with changes in the Gulf Stream system. These new results emerge at a time when results from the Geophysical Fluid Dynamics Laboratory (GFDL) coupled oceanatmosphere model show the existence of similar oscillations in the thermohaline circulation in the North Atlantic with a comparable time scale of 40 to 50 years (Delworth et al., 1993). Deser and Blackmon (1993) also showed a variability of about 10 years. Decadal and interdecadal oscillations driven by a constant flux forcing have been produced in many models (e.g., Huang and Chou, 1994; Huang, 1994; Cai et al., 1995; Cai 1995). In the Cai et al. (1995) study an idealized Atlantic ocean is spun up by restoring the model's uppermost level temperature to a specified temperature climatology alone. When the model reaches a steady state, the forcing is switched to a surface heat flux diagnosed from the steady state. This switch does not induce interdecadal oscillations. A small zonal-redistribution of the diagnosed heat flux then leads to strong interdecadal oscillations of the thermal circulation. By zonal-redistribution, it is meant that the east-west 
variation of the surface heat flux is modified by forming a new flux field that is a linear combination of the diagnosed flux and its zonal mean. In this way, there is no addition or loss of heat associated with the redistribution, and the oceanic heat transport implied by the redistributed flux field is the same as that implied by the diagnosed field. The model SST anomalies are similar to those observed in the North Atlantic (Deser and Blackmon, 1993; Kushnir, 1994), and to those of the interdecadal oscillations in the GFDL coupled atmosphere ocean model (Delworth et al., 1993).

The present study shows that no heat redistribution is required for the generation of oscillations. Diagnosed heat fluxes are not necessarily compatible with the model dynamics. Indeed if the climatology cannot be maintained by the model dynamics, neither will be the diagnosed heat flux. In this context, let us consider the following situations. First, given a surface climatology and an OGCM, an infinite numbers of states and associated heat fluxes may be obtained via different restoration time scales. Second, given an OGCM and a restoration time scale, different flux fields may be obtained with different sets of climatology. Finally, different fluxes may be obtained by changing model parameters. It is just likely that diagnosed fluxes are incompatible with the model dynamics. We show that an incompatibility is readily present if the model domain is extended polewards slightly from that of Cai et al. (1995). This incompatibility has a significant impact even with a small change in the thermal damping strength.

The remainder of this study is divided into three sections. In Sect. 2, the model and the thermal forcing are described. In Sect. 3, model results are presented. The conclusions and the implications for coupled models are given in Sect. 4.

\section{The model and the thermal forcing condition}

This study employs the Pacanowski et al.'s (1991) version of the Bryan-Cox-Semtner OGCM, which is based on the work of Bryan (1969). The horizontal grid spacing is $4^{\circ}$ latitude by $4^{\circ}$ longitude. The model has 12 levels in the vertical, at depths listed in Table 1. Values assigned to the various model parameters are listed in Table 2. These are the same as Cai et al. (1995), Cai and Godfrey (1995), or Cai (1995). The model domain is a flat-bottomed, two-hemisphere Atlantic basin. It has a width of $60^{\circ}$ and a length of $144^{\circ}$ extending from $72^{\circ} \mathrm{S}$ to $72^{\circ} \mathrm{N}$. This extends from the model of Cai et al. (1995) in the north-south direction by one model grid in each model hemisphere. The Southern Hemisphere includes a modeled Antarctic Circumpolar Current (ACC) passage from $44^{\circ} \mathrm{S}$ to $60^{\circ} \mathrm{S}$, and a sill $2350 \mathrm{~m}$ deep in the model's Drake Passage. This sill is intended to provide a realistic ACC transport through the setup of a bottom pressure difference between the two sides of the passage (Holland, 1973; Gill and Bryan, 1971; Cai, 1994). Otherwise the flat bottom would lead to an unrealistically large ACC, and an ACC has to be specified (Cai et al., 1995).
Table 1. Distribution of vertical levels

\begin{tabular}{ccc}
\hline Level & Thickness $(\mathrm{m})$ & Depth of $(T, S)(\mathrm{m})$ \\
\hline 1 & 25.0 & 12.5 \\
2 & 25.0 & 37.5 \\
3 & 40.0 & 70.0 \\
4 & 70.0 & 125.0 \\
5 & 110.0 & 215.0 \\
6 & 200.0 & 370.0 \\
7 & 330.0 & 635.0 \\
8 & 450.0 & 1025.0 \\
9 & 650.0 & 1575.0 \\
10 & 900.0 & 2350.0 \\
11 & 900.0 & 3250.0 \\
12 & 900.0 & 4150.0 \\
\hline
\end{tabular}

Table 2. Values of model coefficients

\begin{tabular}{lll}
\hline Parameter & Symbol & Value \\
\hline Horizontal diffusivity & $A_{\mathrm{TH}}$ & $1 \times 10^{3} \mathrm{~m}^{2} \mathrm{~s}^{-1}$ \\
Horizontal viscosity & $A_{\mathrm{MH}}$ & $3 \times 10^{5} \mathrm{~m}^{2} \mathrm{~s}^{-1}$ \\
Vertical diffusivity & $A_{\mathrm{TV}}$ & $1 \times 10^{-4} \mathrm{~m}^{2} \mathrm{~s}^{-1}$ \\
Vertical viscosity & $A_{\mathrm{MV}}$ & $1 \times 10^{-4} \mathrm{~m}^{2} \mathrm{~s}^{-1}$ \\
\hline
\end{tabular}

Another small modification from that of Cai et al. (1995) is the inclusion of a model Weddell Sea to allow an Antarctic Bottom Water Formation cell.

A simple way in which the incompatibility manifests is through a change in thermal damping. During an ocean spinup, in order to best represent the current ocean climate, strong restorations are usually used. Upon switching to an active atmosphere, the thermal damping weakens, therefore SST in a coupled environment is more free to evolve, and any incompatibility is manifest.

In the present study we use a simple thermal boundary condition. This is diagnosed from the following procedure. Suppose a restoring spinup is carried out in which the model SST is restored to $T_{a}$, and the steady state solution has an SST field $T_{s}$ and a heat flux field $F_{\text {spin }}$. They satisfy

$F_{\text {spin }}=K_{H}\left(T_{a}-T_{s}\right)$.

Here $K_{H}$ is the thermal damping rate during the spinup. The heat flux $Q_{a o}$ is switched to

$Q_{a o}=(1-\alpha) F_{\text {spin }}+\alpha K_{H}\left(T_{a}-T_{1}\right),(\alpha \leq 1)$.

Here $\alpha K_{H}$ is the new damping rate and $T_{1}$ is the timedependent temperature of the uppermost level. At the moment of switching forcing, the heat flux is exactly the same as that implied by the steady-state of the spinup.

The physics behind this treatment of surface thermal forcing can be realized from a simple coupled system consisting of a simple atmosphere and a mixed layer ocean (Cai et al., 1995; Cai and Chu, 1996). The heat balance for the atmosphere is

$0=-K_{H}\left(T_{a}-T_{1}\right)-K_{r}^{\prime} T_{a}+Q_{a}$,

and for the mixed layer ocean is 
$C_{o} \frac{\partial T_{1}}{\partial t}=K_{H}\left(T_{a}-T_{1}\right)+Q_{o}$.

Equation (3) expresses a balance between heat transfer from the ocean to the atmosphere given by the $-K_{H}\left(T_{a}-T_{1}\right)$ term, heat loss to space given by $-K_{r}^{\prime} T_{a}$, and heat sources due to solar input and the divergence of the atmospheric heat transport given by $Q_{a}$. Here, $K_{r}^{\prime}$ is the atmospheric radiative feedback parameter, $C_{o}$ is the specific heat capacity of the mixed layer ocean, and $Q_{o}$ is the oceanic heat source.

Defining a temperature

$T_{r}=Q_{a} / K_{r}^{\prime}$,

we can write Eq. (3) as:

$0=-K_{H}\left(T_{a}-T_{1}\right)+K_{r}^{\prime}\left(T_{r}-T_{a}\right)$.

From Eq. (6), expressing $T_{a}$ in terms of other quantities and substituting into Eq. (4), we have:

$C_{o} \frac{\partial T_{1}}{\partial t}=K_{r}\left(T_{r}-T_{1}\right)+Q_{o}$

where

$K_{r}=\frac{K_{H} K_{r}^{\prime}}{\left(K_{H}+K_{r}^{\prime}\right)}$.

This means that when the mixed layer ocean is coupled to the simple atmosphere described by Eq. (3), its heat balance can be described by Eq. (7). Comparing Eqs. (4) and (7), we see that the heat exchange term

$K_{H}\left(T_{a}-T_{1}\right)=K_{r}\left(T_{r}-T_{1}\right)$.

Defining a ratio

$\alpha=K_{r} / K_{H} \quad(\alpha<1)$,

which measures the change in thermal damping, then we can rewrite the heat exchange term of the simple coupling, using Eqs. (1) and (9), as

$$
\begin{aligned}
K_{r}\left(T_{r}-T_{1}\right) & =K_{r}\left(T_{r}-T_{s}\right)-K_{r}\left(T_{a}-T_{s}\right)+K_{r}\left(T_{a}-T_{1}\right) \\
& =(1-\alpha) F_{\text {spin }}+\alpha K_{H}\left(T_{a}-T_{1}\right) .
\end{aligned}
$$

Thus, the new forcing, which consists of a part of the constant diagnosed heat flux plus a weaker restoring forcing, is equivalent to the simple coupling. Note that when switching from a spinup using Haney restoration as a thermal boundary condition to one provided with a fully coupled model, $\alpha$ changes from 1 to a value ranging from 0.1 to 0.2 . In the present study, we show that an incompatibility can manifest even with a small change in $\alpha$.

\section{Model results}

\subsection{Model spinup}

Initially, the OGCM is spun up by restoring the uppermost level temperature a zonally uniform temperature given by the profile shown in Fig. 1a, which approximates the observed zonally averaged field. This run is referred to as R15 (see Table 3). The profile has
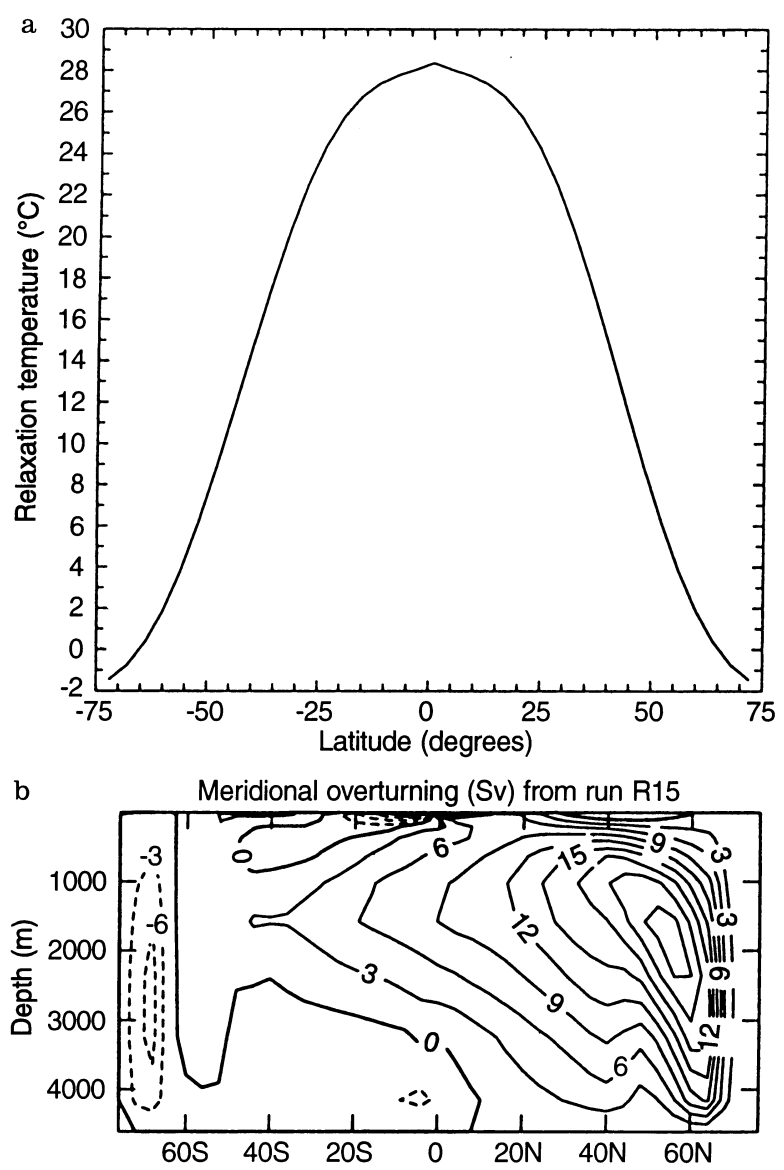

Fig. 1a. Surface relaxation temperature (in ${ }^{\circ} \mathrm{C}$ ), and $\mathbf{b}$ overturning stream function (in Sverdrup) from R15
Table 3. Details of model experiments described in Sect. 3. The second column indicates initial condition. The fourth column shows the forcing condition and the damping time scale is indicated in the brackets. The fifth column indicates whether or not persistent oscillations occur. If yes, the period is given in years $(\mathrm{y})$

\begin{tabular}{lllll}
\hline Run & $\begin{array}{l}\text { Initial } \\
\text { condition }\end{array}$ & $\begin{array}{l}\text { Model } \\
\text { domain }\end{array}$ & Forcing & $\begin{array}{l}\text { Persistent } \\
\text { oscillations }\end{array}$ \\
\hline R15 & Rest & $72^{\circ} \mathrm{S}$ to $72^{\circ} \mathrm{N}$ & Restoring (15 days) & None \\
C30 & R15 & $72^{\circ} \mathrm{S}$ to $72^{\circ} \mathrm{N}$ & New forcing (30 days) & None \\
C45 & R15 & $72^{\circ} \mathrm{S}$ to $72^{\circ} \mathrm{N}$ & New forcing (45 days) & $27.1 \mathrm{y}$ \\
C45r & R15 & $72^{\circ} \mathrm{S}$ to $72^{\circ} \mathrm{N}$ & $\begin{array}{l}\text { Restoring (15 days) } 68^{\circ} \mathrm{N} \\
\text { northward and }\end{array}$ & None \\
& & & New forcing (45 days) & None \\
& & & elsewhere & None \\
R15CGZ & Rest & $68^{\circ} \mathrm{S}$ to $68^{\circ} \mathrm{N}$ & Restoring (15 days) & None \\
CGZ300 & R15CGZ & $68^{\circ} \mathrm{S}$ to $68^{\circ} \mathrm{N}$ & New forcing (300 days) & $24.7 \mathrm{y}$ \\
R45 & R15 & $72^{\circ} \mathrm{S}$ to $72^{\circ} \mathrm{N}$ & Restoring (45 days) & \\
\hline
\end{tabular}


the same analytical expression as in Cai et al. (1995) and the expression applies to the extended model domain, that is, from $68^{\circ}$ to $72^{\circ}$. The $e$-folding restoring time is 15 days. This corresponds to a thermal damping $K_{H}$ of $79 \mathrm{~W} \mathrm{~m}^{-2}{ }^{\circ} \mathrm{C}^{-1}$. The model is also subject to the wind stress used by Bryan (1987). Only during the spinup are the lower level acceleration techniques of Bryan (1984) used. After about 4000 surface years (32000 years at bottom level) of integration, the spinup reaches a statistically steady-state. The overturning circulation is shown in Fig. 1b, and is qualitatively similar to that in the real Atlantic. It features a northern sinking cell; a southern sinking cell; and an Antarctic Circumpolar Current Convective cell. The model ACC reaches $104 \mathrm{~Sv}$.

The convection pattern is shown in Fig. 2. During the spinup, SST is forcefully "nudged" to the prescribed climatology, regardless of whether or not the internal dynamics can maintain it. This nudging in effect prescribes the convection pattern, and the heat flux for its maintenance. Any incompatibility between the assimilated climatology and the model dynamics is suppressed.

\subsection{Drift and variability under the new forcing condition}

In the following two experiments, the thermal damping is switched to the new forcing described by Eq. (2), with a damping rate equivalent to $30(\alpha=0.5)$ and 45 days $(\alpha=0.333)$, respectively. These experiments are referred to as C30 and C45 (see Table 3). The change in thermal damping is small, but this is intended to show the high

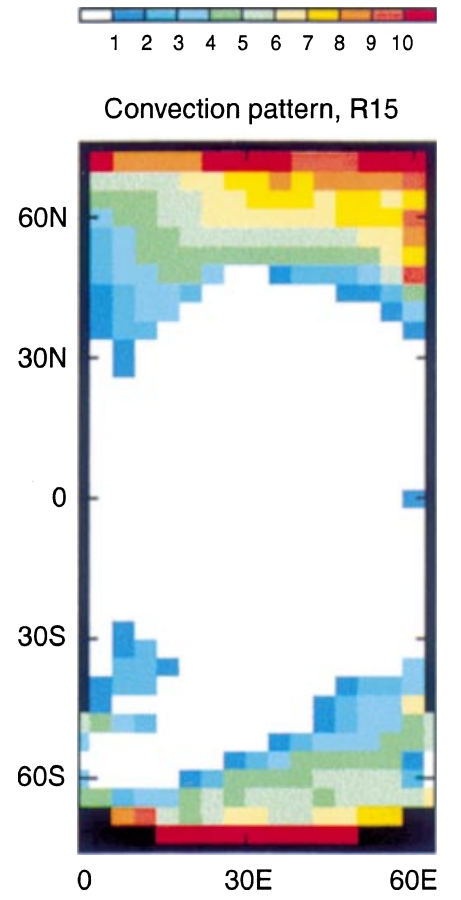

Fig. 2. Convection penetration depth for the run R15. The penetration depth is shown in terms of model levels sensitivity to this parameter in the presence of an incompatibility.

If the model dynamics and the assimilated climatology are fully compatible with each other, then under the new forcing the ocean state should not change, because the new forcing provides an identical air-sea heat flux at the moment of the switching. In the presence of an incompatibility, however, the ocean undergoes significant changes.

Figure 3 shows the time series of the overturning sampled at a location of $\left(60^{\circ} \mathrm{N}, 2350 \mathrm{~m}\right)$. Unless otherwise stated, single-point time series of overturning are all taken at this location. Note that this does not coincide with the location where the overturning under the new forcing is a maximum, nor with the location where the amplitude of the oscillation is a maximum
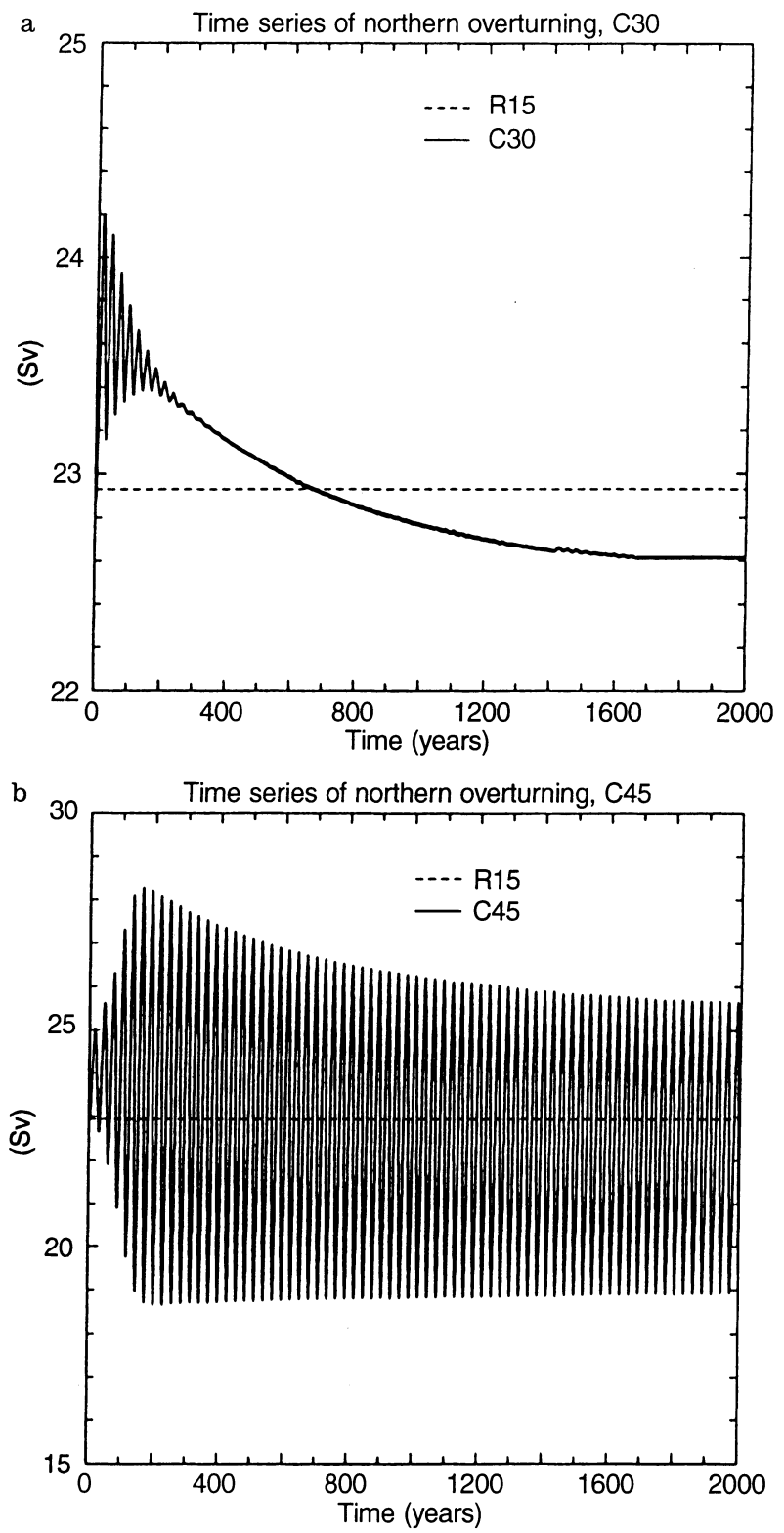

Fig. 3a,b. Time evolution of the northern overturning (in Sverdrup) at a location of $\left(64^{\circ} \mathrm{N}, 2350 \mathrm{~m}\right)$ for a C30 and b C45 
under the flux corrected condition. Several features emerge. First, in run C30 (Fig. 3a), the steady-state drifts from that of R15 with some initial oscillations. The time scale for the drift is several thousands of years. Second, in run C45 (Fig. 3b), besides a similar drift regular thermal oscillations with a period of $27.1 \mathrm{y}$ persist.

To understand the cause for these features, we focus on the results of $\mathrm{C} 45$, since these features are more profound in this case. Figure 4 shows the convection patterns at a quarter period of the steady oscillation. We see that as the overturning oscillates, so does the
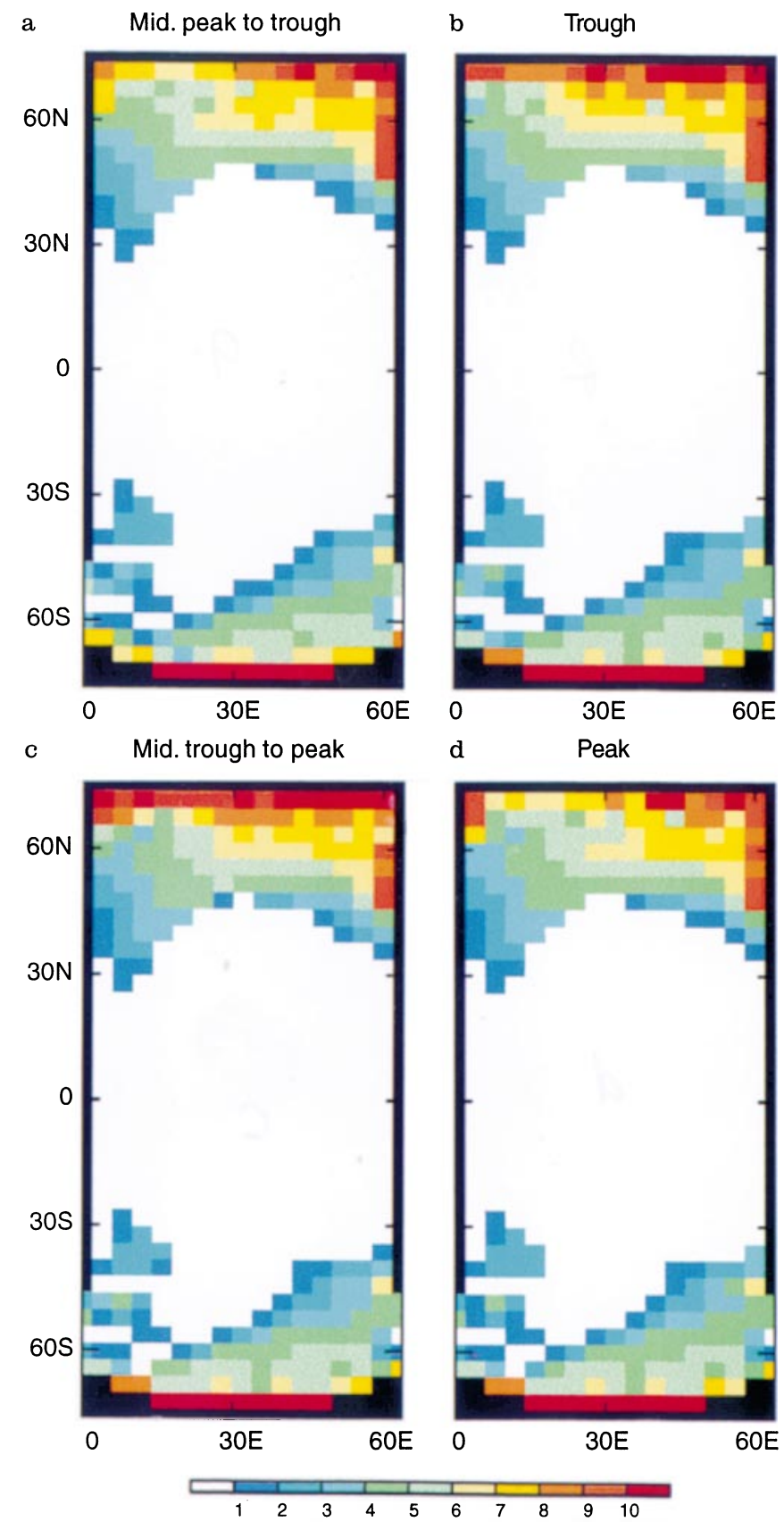

Fig. 4a-d. The same as in Fig. 2 but for run $\mathrm{C} 45$ at a quarter period interval. Panels $\mathbf{b}$ and $\mathbf{d}$ correspond to times when the overturning at the location of $\left(64^{\circ} \mathrm{N}, 2350 \mathrm{~m}\right)$ is at a minimum and maximum respectively convection pattern, especially in the regions $60^{\circ} \mathrm{N}$ northward and off the northeastern boundary. These changes in the convection patterns produce significant drifts of temperatures, especially SSTs, which are shown in Fig. 5, again at a quarter period. We see that drifts at peak and trough are not antisymmetric. It suggests that the mean state has also drifted from the spinup state. Figure $6 \mathrm{a}, \mathrm{b}$ shows the drift of SST and the temperature at $2350 \mathrm{~m}$ of the mean state averaged over 10 steady cycles. At the surface the largest drift occurs in the convection regions, and at depth the ocean is generally cooled. An examination reveals that the cooling is associated with a decrease in the northern high-latitude sinking cell.
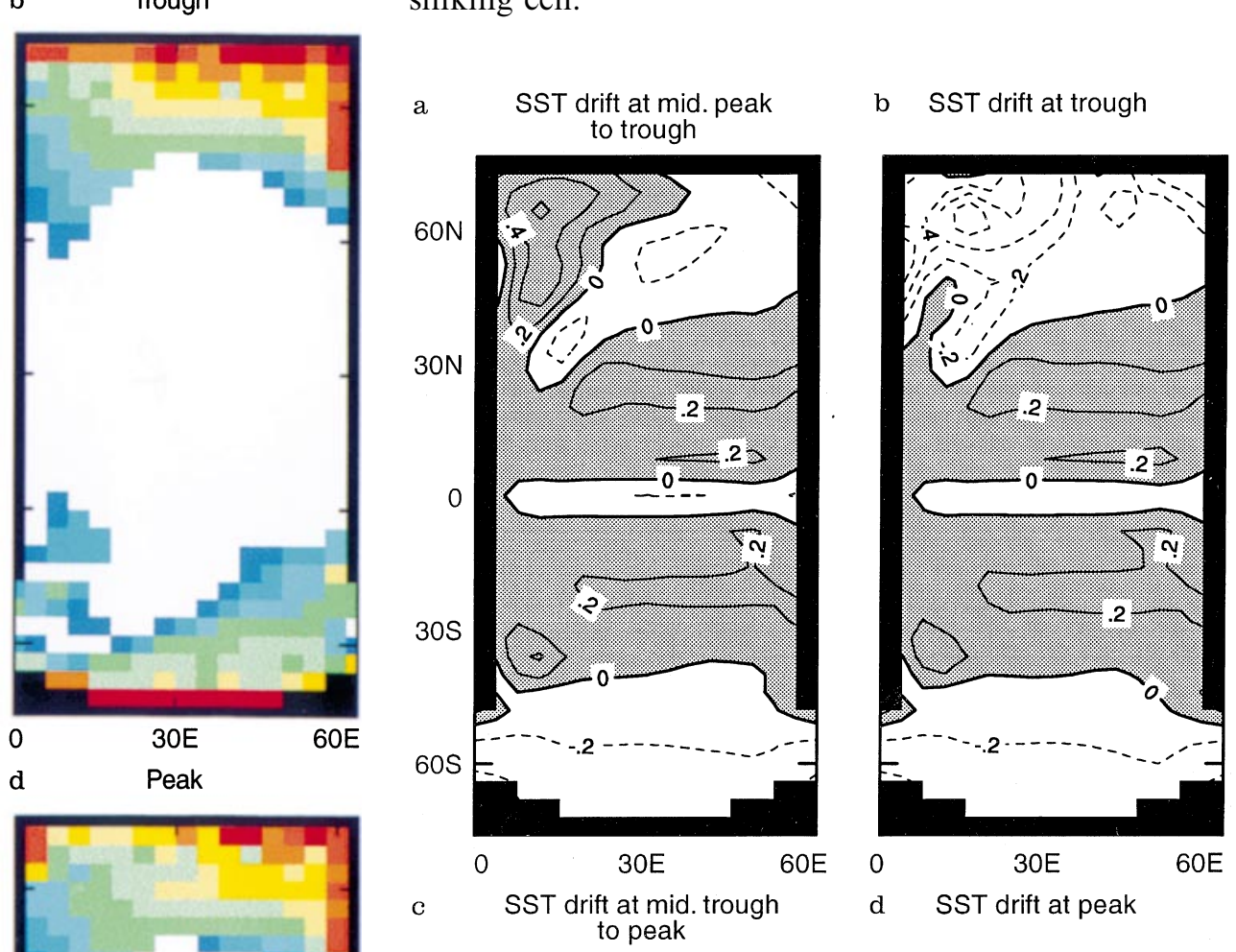

b SST drift at trough
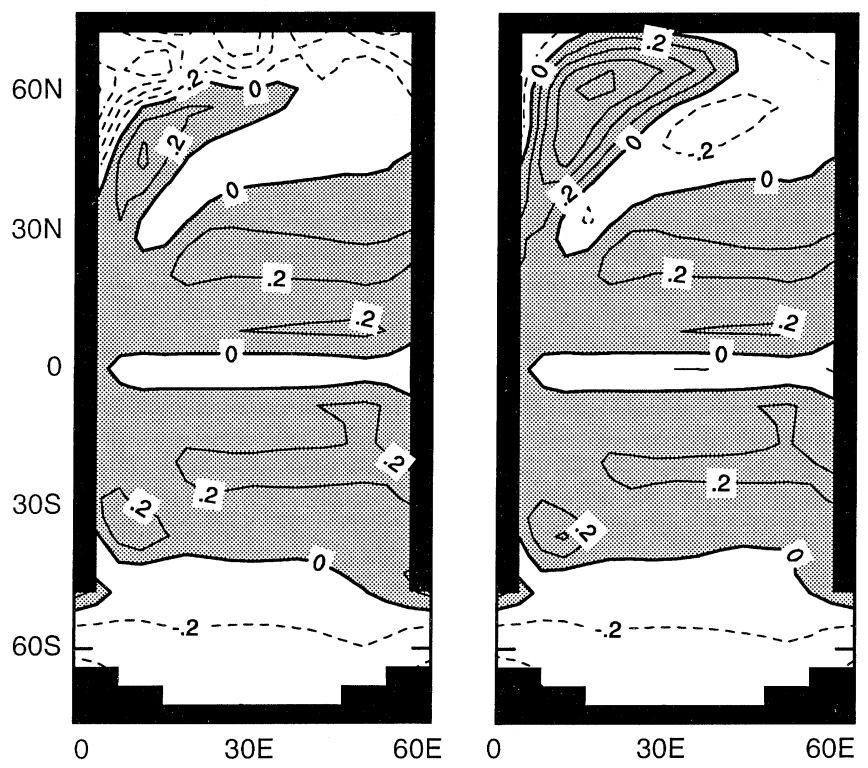

Fig. 5a-d. The same as Fig. 4 but SST drift (in ${ }^{\circ} \mathrm{C}$ ) from the final state of R15 


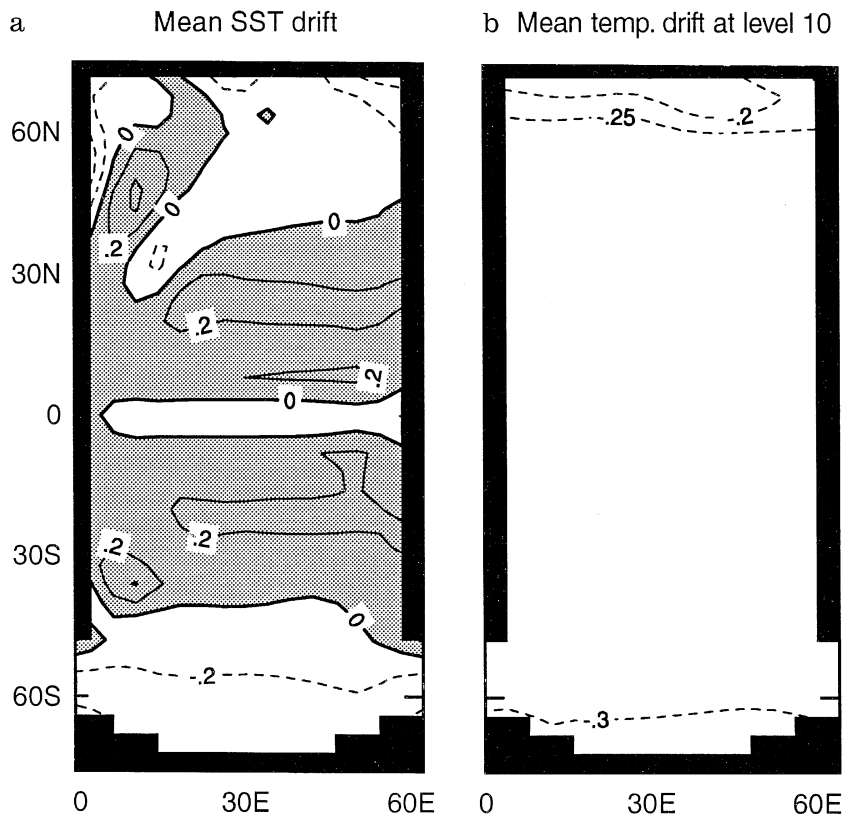

Fig. 6a,b. Drifts of the mean state of $\mathrm{C} 45$ from the steady-state of $\mathrm{R} 15$, a SST and $\mathbf{b}$ temperature at $2350 \mathrm{~m}$. The mean state is obtained by averaging over 10 steady cycles

These features are associated with instability of convections of the spinup state. In the presence of an incompatibility, the equilibrium solution of the spinup via a strong relaxation to a given climatology is not a perfect steady-state solution of the model equations. In areas characterized by sharp oceanic fronts and high convective activity, the model, due to the limitation of model dynamics, cannot maintain the climatology. Therefore, in these areas, the model is trying to systematically move away from the climatology. However under a strong restoration, the surface solution is strongly constrained. Consequently, globally averaged implied surface heat and salinity fluxes (figures not shown) tend to oscillate about some mean value, and convections are intermittent. When the restoration weakens, the surface model field is allowed to evolve so that it is more compatible with the dynamics. The pattern of convections change, and a dynamical adjustment ensues. The features of drift and oscillation are a manifestation of the adjustment process suppressed during the spinup. Since the incompatibility is present permanently, as the dynamical adjustment proceeds, the system is constantly seeking a new convection pattern, giving rise to the oscillation.

The oscillation in the present study is associated with a dynamic adjustment process in a form of waves that propagate along the model basin. Detailed characteristics are discussed in Cai and Chu (1997). In short, the oscillation is associated with generations and propagations of long Planetary waves, that is, westward propogating Rossby waves. This is in sharp contrast to results of Winton (1995) and Greatbatch and Peterson (1996) studies. They show that oscillations in their models are associated with propagations of waves. But they believe that the waves in their models are Kelvin waves, although strictly speaking Kelvin waves are not resolved in OGCMs of their resolutions.

\subsection{Comparison with the results of Cai et al. (1995)}

In order to locate where the incompatibility arises, we compare the present results with those of the Cai et al. (1995) study. Although a similar drift is produced in Cai et al. (1995), no oscillation is induced when the forcing is switched to one with a similar damping rate. As commented upon earlier, the model here extends $4^{\circ}$ poleward (in both hemispheres). The fact that the convection pattern oscillates mainly in this northern extended region suggests that most of the incompatibility arises from this region. To test this point, two more experiments are carried out. Run CGZ300 is conducted in a new model with a north-south extension corresponding exactly to that in Cai et al. (1995), that is, from $68^{\circ} \mathrm{S}$ to $68^{\circ} \mathrm{N}$, and under a new damping corresponding to 300 days. The model experimental procedure and model coefficients are the same as described already involving an initial spinup from rest (R15CGZ, see Table 3). In this experiment, switching to a new damping produces no oscillation, even with a large change in $\alpha$, i.e., from 1 to 0.05 . Figure 7a shows the evolution of the overturning. Although some weak initial oscillations are evident, they die out soon. Run $\mathrm{C} 45 \mathrm{r}$ is identical to $\mathrm{C} 45$, except that in the northern extended region (that is, the row of northernmost grids) the restoring forcing (hence the ' $r$ ') is the same as in the spinup. Figure $7 \mathrm{~b}$ shows the time series of the overturning. Again no oscillation is produced. These two experiments indicate that the incompatibility responsible for the oscillation arises from the extended region. These also suggest that the oscillatory behavior of the convection pattern in other northern high-latitudes in C45 is induced by the incompatibility in the extended region.

\subsection{Results under a weak restoration with no added flux term}

We have argued that the incompatibility between the climatology and the internal model dynamics is responsible for the oscillation in $\mathrm{C} 45$. Such a notion implies that if the incompatibility is not suppressed by the strong restoration as in $\mathrm{R} 15$, it will manifest itself and generate similar oscillations. To this end, R45 is carried out, in which the temperature of uppermost level is relaxed to the climatology (Fig. 1a) with a restoring time of 45 days (see Table 3). This experiment starts from the steady-state of R15, therefore, the only difference from $\mathrm{C} 45$ is that no added flux is employed. At the moment of changing the thermal damping rate, the surface heat flux is suddenly and uniformly reduced to one third of that prior to the change. The reduction of the heat flux initially shocks the system somewhat, but upon recovery the incompatibilityinduced oscillation appears. The period is 24.7 (Fig. 8).

Under this weak restoration, the modeled SSTs drift away from the steady-state of R15o. Figure 9 shows the 

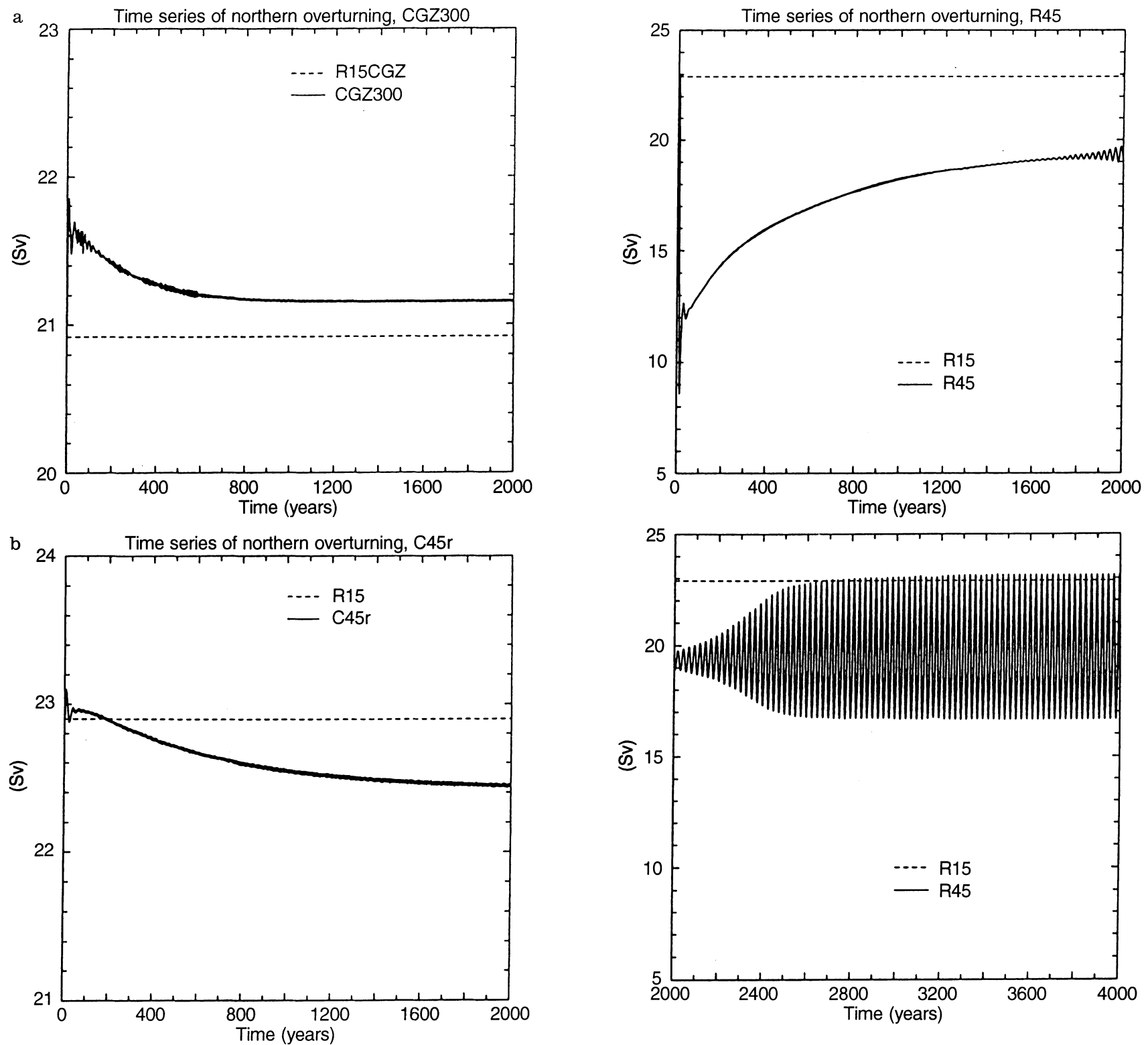

Fig. 7a,b. Time evolution of the northern overturning (in Sverdrup) at a location of $\left(64^{\circ} \mathrm{N}, 23507 \mathrm{~m}\right)$ for a CGZ300 and b C45r. See text for details about these runs

drift of the mean state averaged over 10 steady cycles of the temperature at surface level and at level 10. The surface drift (Fig. 9a) is much larger in comparison with that of C45. In particular, a substantial cooling at tropics and a warming at high latitudes take place, in contrast to the drift under an added flux. The warmer SST is then transmitted to the deep ocean by convection, leading to a general rise in temperature at depth (Fig.9b).

\section{Discussion and summary}

An OGCM is usually spun up via Haney restoration to the present day climatology to an "equilibrium" state. The climatology is forcefully nudged to the model and any incompatibility between the climatology and the

Fig. 8. Time evolution of the northern overturning (in Sverdrup) at a location of $\left(64^{\circ} \mathrm{N}, 2350 \mathrm{~m}\right)$ for $\mathrm{R} 45$

dynamics is suppressed. In this study we demonstrate that when under a weaker thermal damping environment the incompatibility manifests, generating drift (from the spinup) and internal variability. The process is associated with instability of convection, which occurs when the surface forcing changes from a strong restoration to a weaker one. When the thermal damping is weak enough, oscillations occur as a result of a dynamic adjustment process to the permanent presence of the incompatibility. The incompatibility constantly generates perturbations, which propagate as boundary waves along the weakly stratified northern boundary.

It is striking that the generation of oscillations is extremely sensitive to the incompatibility at northernmost grids. A question arises as to how sensitive this result is to the surface climatology used to drive the model. To answer this question, we have carried out 


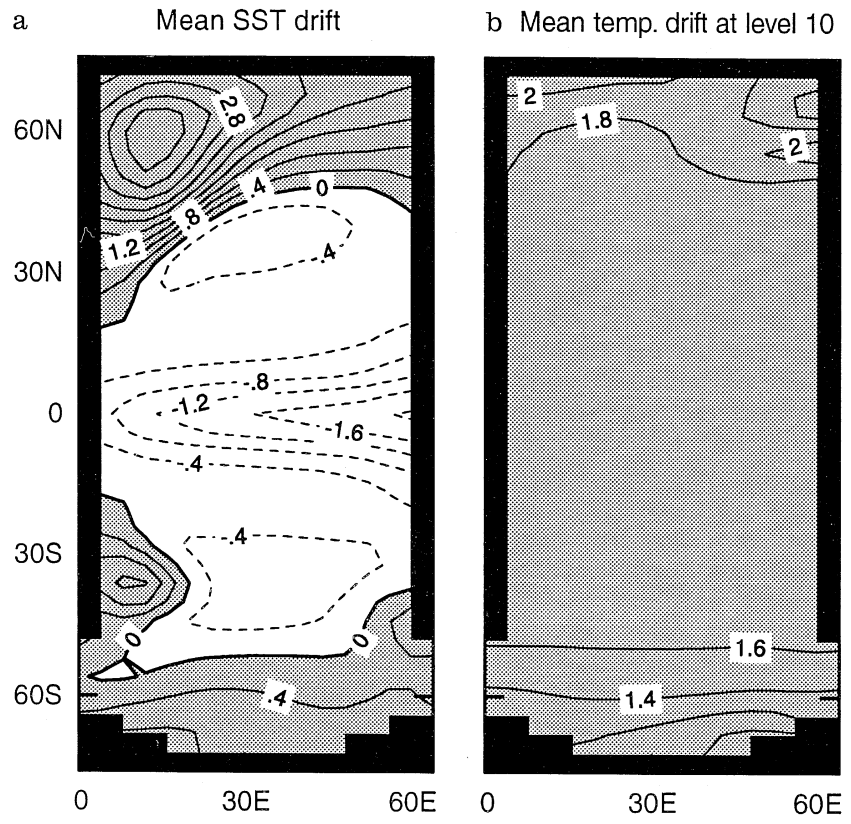

Fig. 9a,b. Drifts of the mean state of R45 from the steady state of $\mathrm{R} 15$, a SST and $\mathbf{b}$ temperature at $2350 \mathrm{~m}$. The mean state is obtained by averaging over 10 steady cycles

numerous experiments varying the north-south gradient of the restoring temperature. In all experiments drift and oscillation occur when the restoring time scale is changed from 15 days to 45 days, suggesting that it may be difficult to achieve a compatibility. Given the fact that instability of a convection pattern plays a significant role in the generation of drift and oscillation, it is highly relevant to ask how sensitive the model result is to the convection scheme used. To address this question, we have repeated run R15 and $\mathrm{C} 45$ using the convection scheme of Rahmstorf (1993). The advantage of the scheme over Cox's (1987) is that it achieves a complete mixing when vertical instability occurs. We found that upon switching to a weaker thermal damping, the model behaves in a similar way to that seen in the original R15o and R45.

That oscillations may be driven by incompatibility between surface forcing and ocean dynamics may have significant implications. On the one hand, in reality, there is no dynamical reason why forcing fields should always be compatible with the oceanic internal dynamics. For example, an atmospheric heat flux is influenced by many atmospheric processes which are weakly dependent on or even completely independent from the ocean (Cai et al., 1995; Cai, 1995). Thus there may exist some incompatibility. In this sense, the incompatibility induced modeled oscillation may simulate a realistic process. On the other hand, there are many factors that can cause an incompatibility during a spinup. These include inaccuracy in the surface climatology, deficiency in model dynamic processes, either absent, poorly parametrized, or not accommodated by the model resolution. For example, at polar latitudes, sea-ice dynamics, which is not included in the present model, may be essential. Another factor that may cause incompatibility is that under polar conditions, especially in the presence of sea-ice, observations are often difficult, and hence a climatology for the region may be poorly constructed.

Pre-coupling spinup of an OGCM via Haney restoration to the present day climatology is commonly carried out to provide initial conditions, and to construct flux adjustment fields (Sausen et al., 1988) for coupled models (e.g., Manabe and Stouffer, 1993, 1994; Gordon and O'Farrell, 1997). Nakamura et al. (1994) show that flux adjustments do not correct errors in climate sensitivity. Marotzke and Stone (1995) confirm this conclusion. Schneider (1996) points out that the manner in which flux adjustment is currently applied is inappropriate in that the adjustment does not eliminate model errors. Our study indicates that in the presence of an incompatibility, a flux adjustment, although designed to remove the systematic mismatch between the atmosphere and the ocean components, has "side effects." One of them is that it will carry the incompatibility to a coupled model, and affect the coupled model behavior. It seems that only when incompatibility is ultimately eliminated from the spinup stage, can flux adjustments truly perform their function without this side effect. To achieve this end, a good climatology and parametrization of model physics are essential.

Acknowledgements. Wenju Cai is supported by the Australian Department of Environment, Sport, and Territories. Peter Chu is supported by the Office of Naval Research High Latitude (HL), Physical Oceanography (PO), and Naval Ocean Modeling and Prediction (NOMP) Programs. Harvey Davies provided the plotting routines for the figures. This work was made possible by the pioneering work of the late Michael Cox, and GFDL for making the MOM code available.

Topical Editor D. J. Webb thanks K. Haines and P. Brasseur for their help in evaluating this paper.

\section{References}

Bryan, K., A numerical method for the study of the circulation of the world ocean, J. Comput. Phys., 4, 347-376, 1969.

Bryan, K., Accelerating the convergence to equilibrium of oceanclimate models, J. Phys. Oceanogr., 14, 666-673, 1984.

Bryan, F., Parameter sensitivity of primitive equation ocean general circulation models, J. Phys. Oceanogr., 17, 970-985, 1987.

Cai, W., Circulation driven by observed surface thermohaline fields in a coarse resolution ocean general circulation model, $J$. Geophys. Res., 99, 10163-10181, 1994.

Cai, W., Interdecadal variability driven by incompatibility between surface flux forcing and oceanic freshwater/heat transport, $J$. Phys. Oceanogr., 25, 2643-2645, 1995.

Cai, W., and P. Chu, Climate drift and interdecadal oscillation due to a change in thermal dumping, J. Clim., 9, 2821-2833, 1996.

Cai, W., and P. Chu, An oscillation under a restorative forcing, $Q$. J. R. Meteorol. Soc., accepted, 1997.

Cai, W., and S. Godfrey, Surface heat flux parametrizations and the variability of thermohaline circulation, J. Geophys. Res., 100, 10679-10692, 1995.

Cai, W., R. J. Greatbatch, and S. Zhang, Interdecadal variability in an ocean model driven by a small, zonal redistribution of the surface buoyancy flux, J. Phys. Oceanogr., 25, 1998-2010, 1995.

Cox, M. D., GFDL Ocean Model Circular No. 7. GFDL/Princeton University, Princeton, N. J., 1 pp., 1987. 
W. Cai, P. C. Chu: Effects of convection instability due to incompatibility between ocean dynamics and surface forcings

Delworth, T., S. Manabe, and R. J. Stouffer, Interdecadal variations of the thermohaline circulation in a coupled oceanatmosphere model, J. Clim., 6, 1933-2011, 1993.

Deser, C., and M. L. Blackmon, Surface climate variations over the North Atlantic Ocean during winter: 1900-1989, J. Clim., 6, 1743-1753, 1993.

Gill, A. E., and K. Bryan, Effects of geometry on the circulation of a three-dimensional southern-hemisphere ocean model, Deep Sea Res., 18, 685-721, 1971.

Gordan, H. B., and S. O'Farrell, Transient climate change in the CSIRO coupled model with dynamics see ice, Mon. Weather Rev., in press, 1997.

Greatbatch, R. J., and K. A. Paterson, Interdecadal variability and oceanic thermohaline adjustment, J. Geophys. Res., 101, $20467-$ 20482, 1996.

Haney, L. R., Surface boundary condition for ocean circulation models, J. Phys. Oceanogr., 1, 241-248, 1971.

Holland, W. R., Baroclinic and topographic influences on the transport in western boundary currents, Geophys. Fluid Dyn., 4, $187-210,1971$.

Huang, R. X., Thermohaline circulation: energetics and variability in a single hemisphere basin model, J. Geophys. Res., 99, 1241112421, 1994.

Huang, R. X., and L. Chou, Parameter sensitivity study of the saline circulation, Clim. Dyn., 9, 391-409, 1994.

Kushnir, Y., Interdecadal variations in North Atlantic sea surface temperature and associated atmospheric conditions, J. Clim., 7, $141-157$.
Manabe, S. and R. J. Stouffer, Century-scale effects of increased atmospheric $\mathrm{CO}_{2}$ on the ocean-atmosphere system, Nature, $\mathbf{3 6 4}$, 215-218, 1993.

Manabe, S. and R. J. Stouffer, Multiple century response of a coupled ocean-atmosphere model to an increase in atmospheric carbon dioxide, J. Clim., 7, 5-23, 1994.

Marotzke, J., and P. Stone, Atmospheric transports, the thermohaline circulation, and flux adjustments in a simple coupled model, J. Phys. Oceanogr., 25, 1350-1364, 1995.

Nakamura, M., P. Stone, and J. Marotzke, Destabilization of the thermohaline circulation by atmospheric eddy transports, $J$. Clim., 7, 1870-1882, 1994.

Pacanowski, R. C., K. W. Dixon, and A. Rosati, GFDL Modular Ocean Model, Users Guide Version 1.0, GFDL Ocean Group Tech. Rep. 2, 46 pp., 1991.

Rahmstorf, S., A fast and complete convection scheme for ocean models, Ocean Model., 101, 9-11, 1993.

Sausen, R., K. Barthel, and K. Hasselmann, Coupled oceanatmosphere models with flux correction, Clim. Dyn., 2, 145163, 1988.

Schneider, E., Flux corrections and the simulation of changing climate, Ann. Geophysicae, 14, 336-341, 1996.

Winton, M., On the role of horizontal boundaries in parameter sensitivity and decadal-scale variability of coarse-resolution ocean general circulation models. J. Phys. Oceanogr., 26, 289304, 1996. 